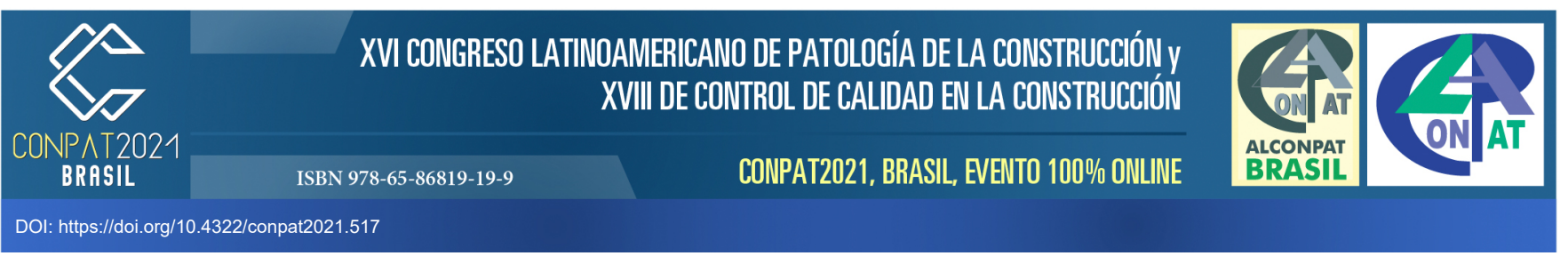

\title{
Aplicação da metodologia GDE/UnB (2015) de inspeção em um edifício residencial de quatro pavimentos.
}

\author{
Teixeira $^{1^{*}}$, Guilherme. Filho ${ }^{1}$, Leonidas. Leonel ${ }^{1}$, Fábio. Silva ${ }^{1}$, Juliano ${ }^{1}$. \\ *Autor de Contacto: guilherme.henrique.eng@hotmail.com \\ ${ }^{1}$ Universidade Estadual de Goiás, Anápolis-GO, Brasil
}

\begin{abstract}
RESUMO
Este trabalho determina o grau de deterioração estrutural de um edifício residencial de quatro pavimentos, executado em concreto armado. A avaliação das manifestações patológicas incidentes na estrutura foi quantificada através da metodologia Grau de Deterioração da Estrutura GDE/UnB (2015). As anomalias contidas nos elementos inspecionados foram registradas através de fotografias, com análise in loco, e, posteriormente, a aplicação da metodologia. Os resultados mostram que o edifício apresentou um grau de deterioração de 55,25, classificando-o como nível alto de deteriorção. Concluiu-se que a aplicação de metodologia GDE/UnB elucidou uma visão geral da condição do objeto de estudo, a qual auxilia durante a inspeção e tomada de decisão, objetivando a correção das falhas existentes e a prevenção de novas deficiências estruturais.
\end{abstract}

Palavras chave: Metodologia GDE; Patologia; Deterioração; Manutenção.

\section{ABSTRACT}


This work determines the degree of structural deterioration of a four-story residential building, built in reinforced concrete. The evaluation of pathological manifestations incident on the structure was quantified using the Degree of Deterioration of the Structure methodology - GDE/UnB (2015). As anomalies contained in the inspected elements were found through photographs, with in loco analysis, and, later, the application of the methodology. The results show that the building has a degree of deterioration of 55.25, classifying it as a high level of deterioration. It was concluded that the application of the GDE/UnB methodology elucidated an overview of the condition of the object of study, which helps during the before and decision making, aiming at correcting existing flaws and preventing new deficiencies before.

Keywords: GDE Methodology; Pathology; Deterioration; Maintenance.

\section{RESUMEN}

Este trabajo determina el grado de deterioro estructural de un edificio residencial de cuatro plantas, ejecutado en hormigón armado. La evaluación de las manifestaciones patológicas incidentes en la estructura fue cuantificada a través de la metodología Grado de Deterioro de la Estructura GDE/UnB (2015). Las anomalías contenidas en los elementos inspeccionados se registraron mediante fotografías, con análisis in situ y, posteriormente, la aplicación de la metodología. Los resultados muestran que el edificio presentaba un grado de deterioro de 55,25, clasificándolo como nivel de deterioro alto. Se concluyó que la aplicación de la metodología GDE/UnB dilucidó una visión general del estado del objeto de estudio, lo que ayuda durante la inspección y la toma de decisiones encaminadas a la corrección de los fallos existentes y la prevención de nuevas deficiencias estructurales.

Palabras clave: Metodología GDE; Patología; Deterioro; Mantenimiento.

\section{INTRODUÇÃO}


A inspeção predial trata-se de uma ferramenta inicial capaz de realizar corretos diagnósticos e se resume na identificação das manifestações patológicas presentes por meio do aspecto visual. (TUTIKIAN; PACHECO, 2013; BOLINA; TUTIKIAN; HELENE, 2019). De modo a auxiliar essa análise e dar maior objetividade, pode-se agregar outras ferramentas como a utilização da metodologia GDE/UnB. Esta metodologia permite atribuir valores numéricos às manifestações patológicas e identificar o estado em que a estrutura se encontra, além de possibilitar uma hierarquização de elementos com prioridade de intervenção.

Segundo Braga (2019), conhecer o estado em que a estrutura se encontra e o seu grau de deterioração é fundamental, pois possibilita o fornecimento de suporte necessário para a busca de soluções de manutenção e reparo.

A metodologia Grau de Deterioração da Estrutura (GDE), da Universidade de Brasília (UnB), foi desenvolvida por Castro (1994) com base nos estudos de Klein et al (1991) em edifícios usuais. Após isso, diversos outros autores trouxeram contribuições para o desenvolvimento da mesma, como por exemplo Lopes (1998), Boldo (2002), Fonseca (2007) e Verly (2015), os quais vieram acrescentando revisões de fatores ou reformulações de determinadas variáveis.

Essa metodologia consiste em, após identificar os elementos deteriorados, dividi-los em famílias de elementos semelhantes e, para cada elemento, atribuir fatores de intensidade (Fi) às manifestações presentes, que tem como finalidade classificar a gravidade em que determinada manifestação se encontra. Da mesma maneira, um fator de ponderação ( $\mathrm{Fp}$ ) é atribuído ao elemento de acordo com a manifestação, pois expressa a importância desse dano para o elemento. Dessa forma, feito isso é possível calcular os parâmetros do grau de dano (D) de cada manifestação patológica e sequencialmente calcular o grau de deterioração do elemento (Gde), grau de deterioração da família (Gdf) e grau de deterioração da estrutura (Gd). Além disso, é possível associar níveis de deterioração e ações recomendadas de acordo com o grau de deterioração.

A aplicação dessa metodologia vem sendo utilizada em diversos trabalhos como ferramenta na análise das manifestações patológicas, buscando maior objetividade. Medeiros et al. (2020) realizaram uma análise das manifestações patológicas presentes em uma ponte situada próximo à cidade de Mossoró-RN e com isso, obteve um grau de deterioração da estrutura (Gd) de 64,03, indicando um nível de deterioração alto. Além disso, realizou-se uma comparação com a metodologia aplicada pelo Departamento Nacional de Infraestrutura e Transportes (DNIT), ao qual conclui-se que a metodologia GDE/UnB apresenta uma maior redução da subjetividade no processo de inspeção.

Lima et al. (2019) realizaram um estudo de caso de manifestações patológicas em três viadutos de Brasília, a fim de quantificar e qualificar esses danos para servirem de suporte na tomada de decisão. Dessa forma, por meio da metodologia foi possível identificar o nível de deterioração de cada viaduto e definir um prazo de intervenção. Além disso, chegaram à conclusão de que o viaduto 02 era o que apresentava maior grau de deterioração da estrutura $(\mathrm{Gd})$, igual a 37,25, valor que foi justificado devido ao maior fluxo de veículos neste viaduto em comparação aos outros. Dessa forma, os autores ressaltam que a metodologia serve para auxiliar o engenheiro na tomada de decisão.

Santana et al. (2019) trabalharam sobre a análise das manifestações patológicas em um edifício, utilizando a metodologia GDE/UnB, Instituto Brasileiro de Avaliações e Perícias de Engenharia (IBAPE) e GUT. O objeto de estudo desta pesquisa sofreu a ação de temperaturas elevadas devido um incêndio. Neste trabalho os autores concluíram que a metodologia GDE/UnB possibilita uma classificação de prioridade com maior clareza do que somente a GUT ou somente a do IBAPE, visto que as análises de vários pontos em ambas metodologias resultaram em coincidência de valor ou classificação.

\section{METODOLOGIA}


A metodologia aplicada neste trabalho consiste na aplicação da metodologia GDE-UnB (2015) para quantificação das manifestações patológicas e identificação da prioridade de intervenção em um prédio residencial de quatro pavimentos, situado na cidade de Anápolis-GO. O edifício nunca passou por processo de intervenção e possui dezesseis anos de idade. Os elementos vistoriados foram divididos em famílias conforme apresentado abaixo.

- Pilares;

- Vigas;

- Lajes;

- Reservatórios;

- Escadas.

Feita essa divisão, realizou-se a atribuição dos fatores de intensidade $\left(\mathrm{F}_{\mathrm{i}}\right)$ e fatores de ponderação $\left(\mathrm{F}_{\mathrm{p}}\right)$ para cada manifestação presente no elemento inspecionado, conforme as tabelas presentes no trabalho de Fonseca (2007). Dessa forma, foi possível realizar o cálculo do grau de dano (D), de acordo com as Equações 1 e 2.

$$
\begin{aligned}
& D=0,8 * F_{p} * F_{i} \quad \quad \text { Para } F_{i} \leq 2,0 \\
& D=\left(12 * F_{i}-28\right) * F_{p} \quad \text { Para } F_{i} \geq 3,0
\end{aligned}
$$

Onde:

D - Grau de dano

$F_{i}$ - Fator de intensidade

$F_{p}$ - Fator de ponderação

Com o grau de dano (D) de cada manifestação, calcula-se o grau de deterioração do elemento $\left(\mathrm{G}_{\mathrm{de}}\right)$ conforme a Equação 3.

$$
G_{d e}=D_{\text {máx }} *\left[1+\frac{\left(\sum_{i=1}^{n} D_{i}\right)-D_{\text {máx }}}{\sum_{i=1}^{n} D_{i}}\right]
$$

Onde:

$G_{d e}$ - Grau de deterioração do elemento;

$D_{i}$ - Grau do dano "i";

$D_{\text {máx }}$ - Maior grau do dano no elemento;

$\mathrm{n}$ - Números de danos no elemento.

Com o valor do grau de deterioração do elemento, relaciona-se um nível de deterioração e assim obtém-se recomendações de ações a serem tomadas. Essa relação pode ser vista na Tabela 1.

Tabela 1 - Classificação do nível de deterioração dos elementos. 
Fonte: (VERLY, 2015) - Modificado.

\begin{tabular}{|c|c|l|}
\hline $\begin{array}{c}\text { Nível de } \\
\text { deterioração }\end{array}$ & $\mathbf{G}_{\text {de }}$ & \multicolumn{1}{c|}{ Ações recomendadas } \\
\hline Baixo & $0-15$ & $\begin{array}{l}\text { Estado aceitável } \\
\text { Manutenção preventiva }\end{array}$ \\
\hline Médio & $15-50$ & $\begin{array}{l}\text { Definir prazo e natureza de nova inspeção } \\
\text { Planejar intervenção em longo prazo (máximo 2 anos). }\end{array}$ \\
\hline Alto & $50-80$ & $\begin{array}{l}\text { Definir prazo para inspeção especializada } \\
\text { Planejar intervenção em médio prazo (máximo 1 ano) }\end{array}$ \\
\hline Sofrível & $80-100$ & $\begin{array}{l}\text { Definir prazo para inspeção especializada rigorosa } \\
\text { Planejar intervenção em curto prazo (máximo 6 meses) }\end{array}$ \\
\hline Crítico & $>100$ & $\begin{array}{l}\text { Intervenção especializada imediata e medidas emergenciais } \\
\text { (alivio de cargas, escoramentos, etc). } \\
\text { Planejar intervenção imediata. }\end{array}$ \\
\hline
\end{tabular}

Para determinar o grau de deterioração dos elementos divididos em grupos semelhantes é calculado o grau de deterioração da família $\left(\mathrm{G}_{\mathrm{df}}\right)$, o qual é função do grau de deterioração do elemento $\left(\mathrm{G}_{\mathrm{de}}\right)$ através da Equação 4.

$$
G_{d f}=G_{d e, \operatorname{máx}} \sqrt{1+\frac{\left(\sum_{i=1}^{n} G_{d e, i}\right)-G_{d e, m a ́ x}}{\sum_{i=1}^{n} G_{d e, i}}}
$$

Onde:

$G_{d e, m a ́ x}$ - Maior grau de deterioração do elemento

$G_{d e, i}$ - Grau de deterioração do elemento "i" ( $\left.\geq 15\right)$

$\mathrm{m}$ - Número de elementos com $G_{d e} \geq 15$

E por fim, ao obter o grau de deterioração de cada família $\left(\mathrm{G}_{\mathrm{df}}\right)$, calcula-se o grau de deterioração da estrutura $\left(\mathrm{G}_{\mathrm{d}}\right)$. A equação utilizada para este cálculo é expressa abaixo, Equação 5.

$$
G_{d}=\frac{K_{m a ́ x}}{7,07} \sqrt{1+\frac{\left(\sum_{i=1}^{k} K_{i}\right)-K_{m a ́ x}}{\sum_{i=1}^{k} K_{i}}}
$$

Onde:

$\mathrm{K}$ - Número de famílias na estrutura

$K_{\text {máx }}$ - Maior valor do produto entre $G_{d e, i}$ pelo respectivo $\mathrm{F}_{\mathrm{r}}$

$K_{i}$-Produto entre $G_{d e, i}$ pelo respectivo $\mathrm{F}_{\mathrm{r}}$

Dessa forma, para o grau de deterioração da estrutura, de maneira semelhante ao grau de deterioração do elemento (Gde), pode ser feita a associação a um nível de deterioração e consequentemente obter medidas que podem ser tomadas quanto essa situação. A tabela aplicada para essa análise trata-se da própria Tabela 1. 


\section{RESULTADOS E DISCUSSÕES}

\subsection{Pilares}

Foram analisados os pilares na região do pilotis, onde foi possível identificar a presença de fissuras e destacamento do concreto devido ao processo de corrosão da armadura. Trata-se de uma região exposta ao sol, chuva e uma grande quantidade de dióxido de carbono. Sendo identificado através do ensaio de aspersão de fenolftaleína que o processo de corrosão ocorre devido à carbonatação. Dentre os pilares, aqueles que apresentaram aspectos visuais de danos foram o P05, P06, P19, P30, P31, P38, P46 e P47.

Os pilares P05, P06, P19, P38, P46 e P47, como pode ser visto na Figura 1, apresentam o desenvolvimento de corrosão ainda em estágio inicial. Isso é devido à falta de manchas e de fissuras de corrosão e o desplacamento que acontece nos elementos é superficial, na forma de escamação. Por esse motivo, adotou-se o fator de intensidade $\left(\mathrm{F}_{\mathrm{i}}\right)$ igual a 2 tanto para as fissuras quanto para $\mathrm{o}$ destacamento do concreto. Além disso, segundo Castro (1994), não é necessário considerar a carbonatação visto que já ocorre a corrosão. Para evitar superposição considera-se a intensidade apenas da corrosão.
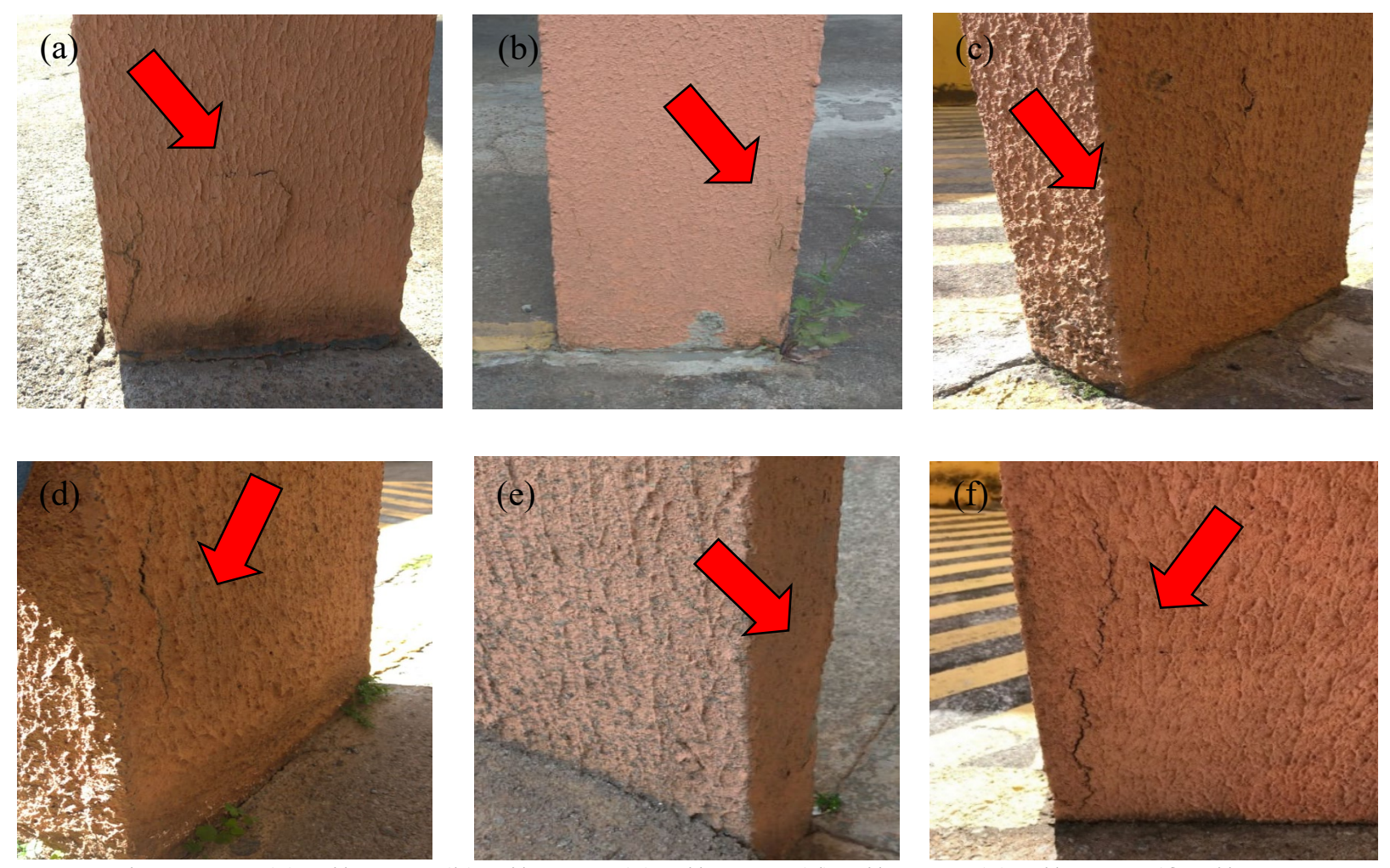

Figura 1 - (a) Pilar 05, (b) Pilar 06, (c) Pilar 19, (d) Pilar 38, (e) Pilar 46, (f) Pilar 47.

Dessa forma, o Grau de deterioração do elemento $\left(\mathrm{G}_{\mathrm{de}}\right)$ para cada um dos pilares citados acima é abaixo de 15, o que representa um nível de deterioração baixo, ficando em um estado aceitável, necessitando apenas de manutenções preventivas. O cálculo realizado apresenta-se na Tabela 2.

Tabela 2 - Grau de deterioração dos pilares P05, P06, P19, P38, P46, P47. 


\begin{tabular}{|c|c|c|c|}
\hline Nome do elemento & \multicolumn{3}{|c|}{ P05 $=\mathbf{P 0 6}=\mathbf{P 1 9}=\mathbf{P 3 8}=\mathbf{P 4 6}=\mathbf{P 4 7}$} \\
\hline Local & \multicolumn{3}{|c|}{ Pilotis } \\
\hline Danos & Fp & Fi & D \\
\hline Corrosão & 5 & 2 & 8 \\
\hline Desplacamento & 3 & 2 & 4,8 \\
\hline \multirow{3}{*}{$\mathrm{G}_{\mathrm{de}}$} & 11 \\
\cline { 2 - 4 }
\end{tabular}

O pilar 30, Figura 2, apresenta um processo avançado de corrosão, porém não foi possível observar a perda de seção da armadura. Por isso, foi considerado um fator de intensidade da corrosão igual a 3 e para o desplacamento, por ocorrer um lascamento capaz de expor a armadura em grandes proporções, o fator de intensidade considerado é igual a 3. Com isso, o grau de deterioração do elemento é igual a 55, conforme a Tabela 3. Dessa forma, constata-se que o elemento apresenta um nível de deterioração alto, caracterizando a necessidade de inspeções especializadas e necessidade de intervenções em médio prazo (máximo 1 ano).

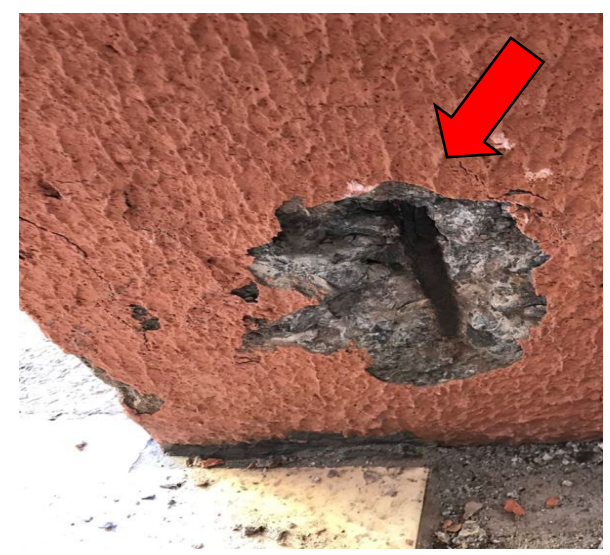

Tabela 3 - Grau de deterioração do pilar P30.

\begin{tabular}{|c|c|c|c|}
\hline Nome do elemento & \multicolumn{3}{|c|}{ P30 } \\
\hline Local & \multicolumn{3}{|c|}{ Pilotis } \\
\hline Danos & Fp & Fi & D \\
\hline Corrosão & 5 & 3 & 40 \\
\hline Desplacamento & 3 & 3 & 24 \\
\hline \multirow{2}{*}{$\mathrm{G}_{\mathrm{de}}$} & 55 \\
\hline
\end{tabular}

Figura 2 - Pilar 30.

O pilar 31, Figura 3, não apresenta o desplacamento da seção de concreto, porém ocorre um processo de corrosão identificado pela presença de fissuras paralelas as armaduras, caracterizando um fator de intensidade 3. Dessa forma, o grau de deterioração do elemento é de 40, conforme a Tabela 4, caracterizando um nível de deterioração médio, o qual necessita de intervenção de no máximo 2 anos.

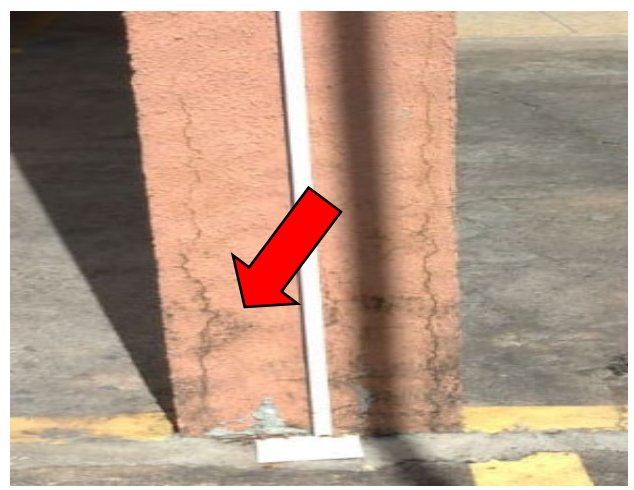

Tabela 4 - Grau de deterioração do pilar P31.

\begin{tabular}{|c|c|c|c|}
\hline Nome do elemento & \multicolumn{3}{|c|}{ P30 } \\
\hline Local & \multicolumn{3}{|c|}{ Pilotis } \\
\hline Danos & Fp & Fi & D \\
\hline Corrosão & 5 & 3 & 40 \\
\hline Desplacamento & 3 & 0 & 0 \\
\hline \multicolumn{3}{|c|}{$\mathrm{G}_{\mathrm{de}}$} & 40 \\
\hline
\end{tabular}

Figura 3 - Pilar 31. 
Para o cálculo do grau de deterioração da família $\left(\mathrm{G}_{\mathrm{df}}\right)$ dos pilares foi utilizado apenas os valores resultantes dos pilares P30 e P31, visto que, segundo a metodologia, os valores de $\mathrm{G}_{\mathrm{de}}$ abaixo de 15 não devem ser considerados nos cálculos, pois não são representativos. Com isso, o valor do grau de deterioração da família $\left(\mathrm{G}_{\mathrm{df}}\right)$ foi de 65,56 , conforme a Tabela 5 .

Tabela 5 - Grau de deterioração da família de pilares.

\begin{tabular}{|c|c|c|}
\hline Nome do elemento & $\mathbf{G}_{\mathbf{d e}}$ & $\mathbf{G}_{\mathbf{d f}}$ \\
\cline { 1 - 2 } P30 & 55 & \multirow{2}{*}{65,56} \\
\hline P31 & 40 & \\
\hline
\end{tabular}

\subsection{Lajes}

Foram identificados danos nas lajes em três regiões. O primeiro dano (L01) na região dos pilotis, logo abaixo da região do banheiro de um dos apartamentos. O segundo dano (L02) na sacada do apartamento, situada na parte à esquerda da fachada frontal. O terceiro dano (L03) lozalizado no terceiro pavimento, na parte inferior da laje de cobertura, na região do reservatório. A Figura 4 mostra as lajes danificadas.
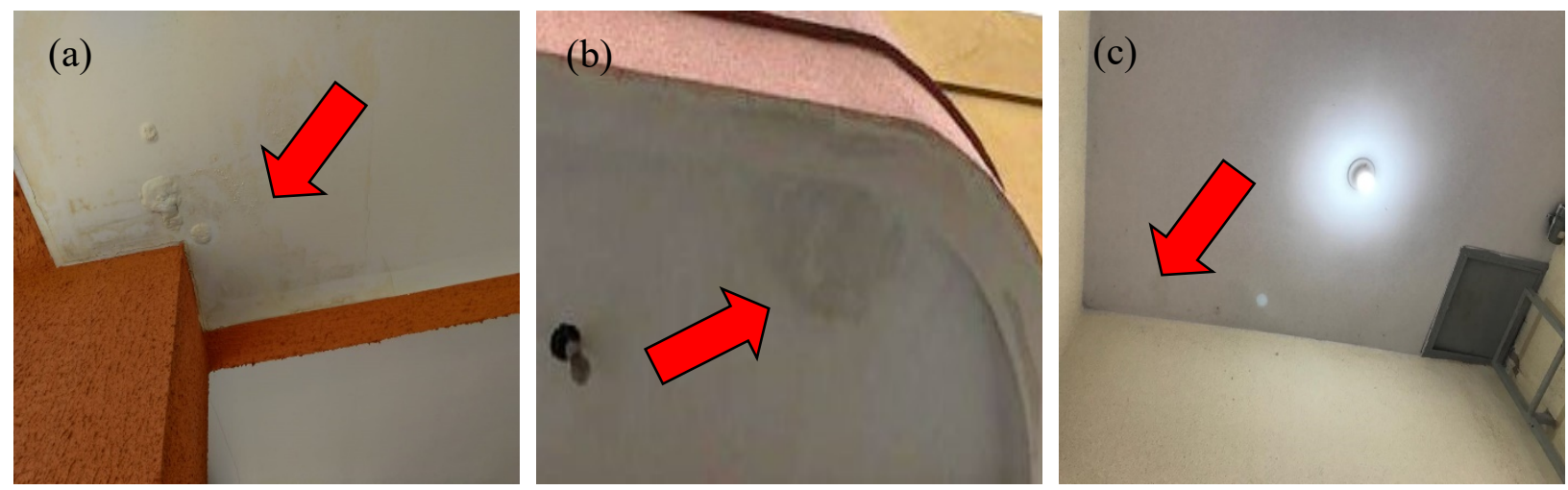

Figura 4 - (a) Laje L01, (b) Laje L02, (c) Laje L03.

As manifestações identificadas são relacionadas com a presença de umidade, sendo considerados fatores de intensidade de 1 e 2, como pode ser visto nas Tabelas 6 e 7. Porém, não representam danos significativos para a estrutura, uma vez que apresentam um nível de deterioração baixo, enquadrando-se em um estado aceitável que necessita apenas de manutenções preventivas. Dessa maneira, como os elementos identificados apresentam valores de grau de deterioração $\left(\mathrm{G}_{\mathrm{de}}\right)$ inferiores a 15, conforme Castro (1994), os mesmos não são representativos para considerações de cálculo do grau de deterioração da família $\left(\mathrm{G}_{\mathrm{df}}\right)$. Dessa forma, o valor de $\mathrm{G}_{\mathrm{df}}$ será igual a 0 .

Tabela 6 - Grau de deterioração da laje L01 Tabela 7 - Grau de deterioração da laje L03. e L02.

\begin{tabular}{|c|c|c|c|}
\hline Nome do elemento & \multicolumn{4}{|c|}{ L01=L02 } \\
\hline Local & \multicolumn{3}{|c|}{ Pilotis/Sacada } \\
\hline Danos & Fp & Fi & D \\
\hline Umidade & 3 & 2 & 4,8 \\
\hline \multicolumn{3}{|c|}{$\mathrm{G}_{\mathrm{de}}$} & 4,8 \\
\hline
\end{tabular}

\begin{tabular}{|c|c|c|c|}
\hline Nome do elemento & \multicolumn{3}{|c|}{ L03 } \\
\hline Local & \multicolumn{3}{|c|}{$3^{\circ}$ Pavimento } \\
\hline Danos & Fp & $\mathbf{F i}$ & D \\
\hline \multirow[t]{2}{*}{ Umidade } & 3 & 1 & 2,4 \\
\hline & \multicolumn{2}{|c|}{$\mathrm{G}_{\mathrm{de}}$} & 2,4 \\
\hline
\end{tabular}




\subsection{Vigas}

As vigas vistoriadas não apresentaram nenhuma anomalia visível.

\subsection{Escada}

Na escada (E01), região do primeiro lance, foi observado o destacamento do concreto e início do processo corrosivo, como pode ser observado na Figura 5. Porém, como não foi possível identificar com clareza a perda de seção da armadura, considerou-se um fator de intensidade igual a 3 para o processo corrosivo. Quanto ao destacamento de concreto, devido à constatação de um processo já em fase de exposição de armadura, o valor considerado para o fator de intensidade foi igual a 3. Além disso, esses tipos de manifestações para as escadas representam fatores de ponderação igual a 5 para a corrosão e igual a 3 para o destacamento, logo o grau de deterioração do elemento é de 55 , conforme Tabela 8 . Dessa forma, constata-se que o elemento apresenta nível de deterioração alto, caracterizando uma necessidade de inspeções especializadas e intervenção dentro de um prazo médio (máximo de 1 ano).

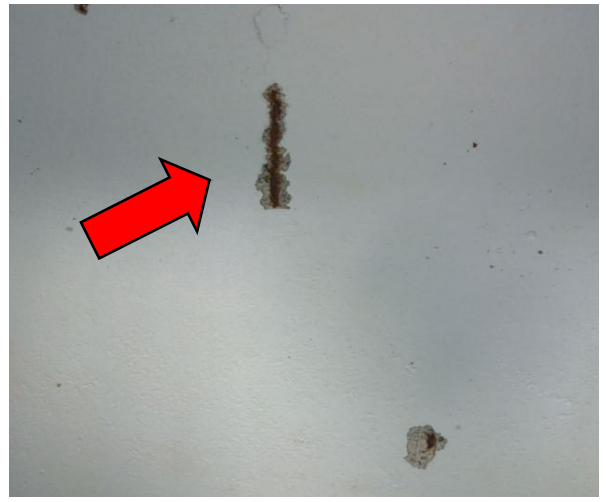

Figura 5 - Laje E01.

Tabela 8 - Grau de deterioração da laje E01.

\begin{tabular}{|c|c|c|c|}
\hline Nome do elemento & \multicolumn{4}{|c|}{ L01 } \\
\hline Local & \multicolumn{3}{|c|}{ Térreo } \\
\hline Danos & Fp & Fi & D \\
\hline Corrosão & 5 & 3 & 40 \\
\hline Desplacamento & 3 & 3 & 24 \\
\hline \multirow{2}{*}{ Gde $_{\text {de }}$} & 55 \\
\cline { 2 - 4 }
\end{tabular}

Como possui apenas um elemento dentro da família, o valor do grau de deterioração da família $\left(\mathrm{G}_{\mathrm{df}}\right)$ será igual a 55 .

\subsection{Reservatório}

O reservatório superior (R1), mostrado na Figura 07, apresenta fissuras devido movimentações higrotérmicas e manchas escuras devido ao acúmulo de umidade. Por esse motivo, atribuiu-se um fator de intensidade 3 pela impermeabilização deficiente em alguns pontos, identificadas a partir das manchas escuras, o fator de ponderação para esta manifestação é igual a 4. Para as fissuras, por conta das pequenas dimensões e por apresentarem sinais de estabilização, considerou-se um valor de fator de intensidade igual a 2 e o fator de ponderação para essa manifestação igual a 3. Logo, o grau de deterioração do elemento é de 36,17, conforme Tabela 08. Dessa forma, o elemento apresenta um nível de deterioração médio, sendo necessário definir intervenções em longo prazo (máximo de 2 anos). Já o reservatório inferior (R2) não apresenta sinais de deterioração. Necessitando apenas de se manter a periodicidade das manutenções preventivas.

Como apenas o elemento R1 é determinante para o cálculo do grau de deterioração da família, o seu valor será de 36,17 . 


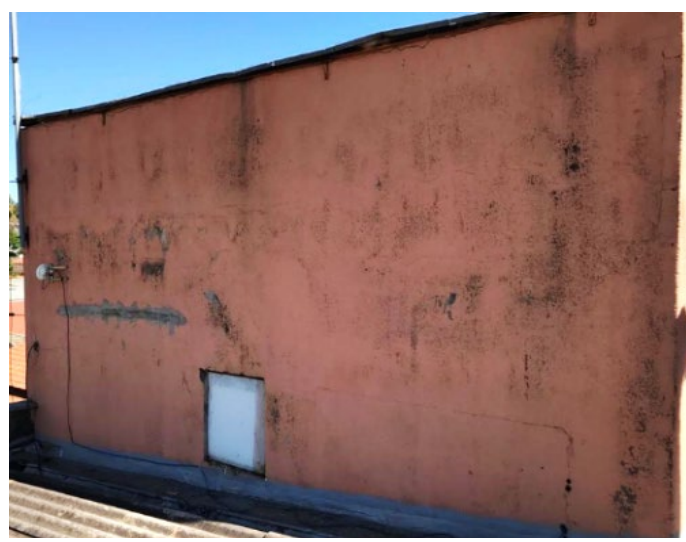

Tabela 9 - Grau de deterioração da laje R01.

Figura 6 - Paredes R01.

\begin{tabular}{|c|c|c|c|}
\hline Nome do elemento & \multicolumn{4}{|c|}{ R01 } \\
\hline Local & \multicolumn{3}{|c|}{ Reservatório } \\
\hline Danos & Fp & Fi & D \\
\hline Fissuras & 3 & 2 & 4,8 \\
\hline $\begin{array}{c}\text { Impermeabilização } \\
\text { deficiente }\end{array}$ & 4 & 3 & 32 \\
\hline \multirow{2}{*}{$\mathrm{G}_{\mathrm{de}}$} & 36,17 \\
\cline { 2 - 4 }
\end{tabular}

\subsection{Grau de deterioração da estrutura}

Por fim, com o grau de deterioração de cada família associado ao seu respectivo fator de relevância, o grau de deterioração da estrutura obtido foi de 55,25, como pode ser observado por meio da Tabela 10. Logo, conclui-se que o edifício se apresenta em um nível de deterioração alto, o que requer uma inspeção especializada e intervenção dentro de um prazo médio (máximo de 1 ano). Dessa forma, analisando os elementos deteriorados e classificando quanto a prioridade, temos que a família de pilares é a que mais necessita de atenção, seguidos da escada e posteriormente do reservatório, como pode ser visto na Tabela 10. Obtemos, dentre todos os elementos, a hierarquização de prioridade apresentada na Tabela 11.

Tabela 10 - Grau de deterioração da estrutura.

\begin{tabular}{|c|c|c|c|}
\hline Família & $\mathbf{G}_{\mathbf{d f}}$ & $\mathbf{F r}$ & \multirow{2}{*}{$\mathbf{G}_{\mathbf{d}}$} \\
\hline Pilares & 65,56 & 5 & \multirow{2}{*}{5} \\
\cline { 1 - 3 } Vigas & 0 & 5 & \multirow{2}{*}{55,25} \\
\cline { 1 - 3 } Lajes & 0 & 4 & \\
\hline Escada & 55 & 3 & \\
\hline Reservatório & 36,17 & 2 & \\
\hline
\end{tabular}

Tabela 11 - Grau de deterioração dos elementos.

\begin{tabular}{|c|c|c|c|}
\hline Elemento & $\mathbf{G}_{\mathbf{d e}}$ & Nível de deterioração & Prazo \\
\hline P30 & 55,00 & Alto & 1 ano \\
\hline E01 & 55,00 & Alto & 2 anos \\
\hline P31 & 40,00 & Médio & 2 anos \\
\hline R01 & 36,17 & Médio & Manutenção preventiva \\
\hline P05 & 11,00 & Baixo & Manutenção preventiva \\
\hline P06 & 11,00 & Baixo & Manutenção preventiva \\
\hline P19 & 11,00 & Baixo & Manutenção preventiva \\
\hline P38 & 11,00 & Baixo & Manutenção preventiva \\
\hline P46 & 11,00 & Baixo & Manutenção preventiva \\
\hline P47 & 11,00 & Baixo & Manutenção preventiva \\
\hline L01 & 4,80 & Baixo & Manutenção preventiva \\
\hline L02 & 4,80 & Baixo & Manutenção preventiva \\
\hline L03 & 2,40 & Baixo & \\
\hline
\end{tabular}




\section{CONCLUSÃO}

Com base na realização deste trabalho foi possível concluir que com a aplicação de metodologia GDE/UnB (2015) obtém-se uma visão geral da condição da edificação, a qual auxilia durante a inspeção e na tomada de decisão, fornecendo informações necessárias para identificar pontos que necessitam de maior atenção. Além disso, por meio da mesma, observa-se uma diminuição da subjetividade na análise e determinação dos pontos de maior prioridade devido à aplicação dos fatores e formulações matemáticas.

No edifício de estudo, por meio da aplicação da metodologia, identificou-se que os pontos mais críticos tratavam-se da região dos pilares e da escada, dos quais os elementos P30 e E01 possuíam, ambos, grau de deterioração igual a 55, o que representa um nível de deterioração alto e sugestão de prazo de intervenção de no máximo 1 ano, demonstrando uma urgência maior.

Com nível médio de deterioração encontra-se o pilar P31 e o reservatório superior, R01, respectivamente, com o grau de deterioração igual a 40 e 36,17, exigindo que seja realizada uma intervenção em um prazo máximo de 2 anos.

Os demais elementos vistoriados possuem um nível de deterioração baixo, mostrando um estado aceitavael, sendo necessário apenas a realização de manutenções preventivas.

Dessa forma, o edifício como um todo apresenta grau de deterioração igual a 55,25 e se enquadra em um nível alto de deterioração, o que requer intervenção dentro de um prazo máximo de 1 ano. Com essas informações e atrelada com a realização da inspeção e diagnostico é possível direcionar o engenheiro a uma decisão mais objetiva e concentrando os esforços nos pontos de maior prioridade.

\section{REFERÊNCIAS}

Tutikian, B., Pacheco, M. (2013), “Boletim Técnico $n^{\circ} 1$-Inspeção, Diagnóstico e Prognóstico na Construção Civil”, ALCONPAT, México, Mérida, p. 17

Bolina, F., Tutikian, B., Helene, P. (2019), "Patologia de Estruturas”, Oficina do Texto, Brasil, São Paulo, p. 320

Boldo, P. (2002), "Avaliação quantitativa de estruturas de concreto armado de edificações no âmbito do Exército Brasileiro”, Dissertação de Mestrado, Universidade de Brasília, 312 p.

Braga et.al., (2019), Aplicação da Matriz GUT na análise de manifestações patológicas em construções históricas. Revista ALCONPAT. 9 (3): 320-335. https://doi.org/10.21041/ra.v9i3.400

Castro, E. (1994), “Desenvolvimento de metodologia para manutenção de estruturas de concreto armado”, Dissertação de Mestrado, Universidade de Brasília, 139 p.

Fonseca, R. (2007), “A estrutura do Instituto Central de Ciências: Aspectos históricos, científicos e tecnológicos de projeto, execução, intervenções e proposta de manutenção”, Dissertação de Mestrado, Universidade de Brasília, 231 p.

Klein et. al., (1991), “Critérios adotados na vistoria e avaliação de obras de arte” em: XXV Jornadas Sul-Americanas de Engenharia Estrutural, Porto Alegre: UFRGS, pp. 185-197.

Lima et.al., (2019), Análise de manifestações patológicas do concreto em viadutos urbanos. Revista ALCONPAT. 9 (2): 247-259. https://doi.org/10.21041/ra.v9i2.308 
Lopes, B. (1998), "Sistema de manutenção predial para grandes estoques de edifícios: Estudo para inclusão do componente "Estrutura de Concreto", , Dissertação de Mestrado, Universidade de Brasília, 158 p.

Medeiros et.al., (2020), Aplicação de metodologias de inspeção em ponte de concreto armado. Ambiente Construído. 20 (3): 687-702. https://doi.org/10.1590/s1678-86212020000300453

Santana et.al., (2019), "Manifestações patológicas em estruturas de concreto armado submetidas a ação de incêndio” em: Congresso Brasileiro de Concreto, Fortaleza: IBRACON, 17 p.

Verly, R. (2015), “Avaliação de metodologias de inspeção como instrumento de priorização de intervenções em obras de arte especiais”, Dissertação de Mestrado, Universidade de Brasília, 198 p. 